\section{Automatic Generation of Landmark-based Indoor Routing Instructions}

Gl_Forum 2017, Issue 1

Page: $106-113$

Short Paper

Corresponding Author:

irene.fellner@wu.ac.at

DOI: 10.1553/giscience2017_01_s106

Irene Fellner

IT-Services, Vienna University of Economics and Business, Austria

\begin{abstract}
This paper addresses the question of how route instructions that include landmarks for indoor navigation can be created automatically based on information available in spatial indoor databases. Existing approaches for the generation of landmark-based routing instructions are designed for outdoor environments and rely mainly on instance-level data about the characteristics of potential landmarks. However, indoor navigation differs from outdoor navigation in various aspects, and indoor spatial databases do not usually contain detailed information about individual objects. Therefore, the proposed method aims to address the specific challenges of indoor navigation and relies only on commonly available data about categories of spatial objects. A case study of a university campus demonstrates that by and large the method produces correct routing instructions that include landmarks. However, it also reveals some gaps in the model and shows possibilities for enhancements for future work.
\end{abstract}

\title{
Keywords:
}

indoor navigation, landmarks, routing instructions

\section{Introduction}

Landmarks play an important role in spatial recognition and learning new environments (Siegel \& White, 1975). Sorrows \& Hirtle (1999) define landmarks as prominent, identifying features in an environment that can be easily recognized and memorized. Although landmarks are important to enrich route descriptions, for example to help identify where a left or right turn should be made (Lovelace et al., 1999; Rehrl et al., 2009; Tom \& Denis, 2003), there are hardly any pedestrian navigation systems that include landmarks in their route instructions. The reason for this is the absence of reliable methods to identify landmarks and integrate them automatically into routing instructions.

Current approaches concerning the integration of landmarks into routing instructions are mainly limited to outdoor environments and to the extraction of landmarks based on detailed visual or geometric information about spatial objects (Elias, 2003; Raubal \& Winter, 2002). While for outdoor environments this information may be available, for example in the 
form of geo-referenced images or digital cadastral maps, it is not usually available for indoor objects.

An alternative approach was proposed by Duckham, Winter and Robinson (2010). They developed a 'landmark navigation model' (LNM) which incorporates landmarks into route instructions and relies solely on class-level information about types of landmarks, instead of using specific instance-level data about the visual or geometric characteristics of individual features.

The LNM is designed for outdoor navigation. As navigation within buildings has special characteristics and challenges in contrast to outdoor navigation, e.g. in terms of landmark characteristics, routing networks and routing instructions, the model cannot be directly applied to indoor navigation.

This paper presents an 'indoor landmark navigation model' (ILNM) for use within buildings and for the automatic selection of landmarks from different feature types found within a spatial indoor database. The ILNM is based on the methods and algorithm of the LNM created by Duckham et al. (2010) but is adjusted to the special aspects of indoor navigation.

In order to evaluate the ILNM for its feasibility, it is applied to the data of the Geographic Information System (GIS) of the Vienna University of Economics and Business (WU), the 'WU Campus GIS'.

This paper is based on work presented also in Fellner (2015).

\section{Indoor Landmark Navigation Model (ILNM)}

\section{Overview}

The 'indoor landmark navigation model' (ILNM) provides a method for the automatic creation of route instructions with landmarks for indoor environments. The model consists of three main steps, which are illustrated in Figure 1:

1. Landmark identification: Identification of suitable landmark candidates

2. Landmark selection: Selection of landmarks for an individual route

3. Landmark integration: Integration of selected landmarks into route instructions for a specific route 


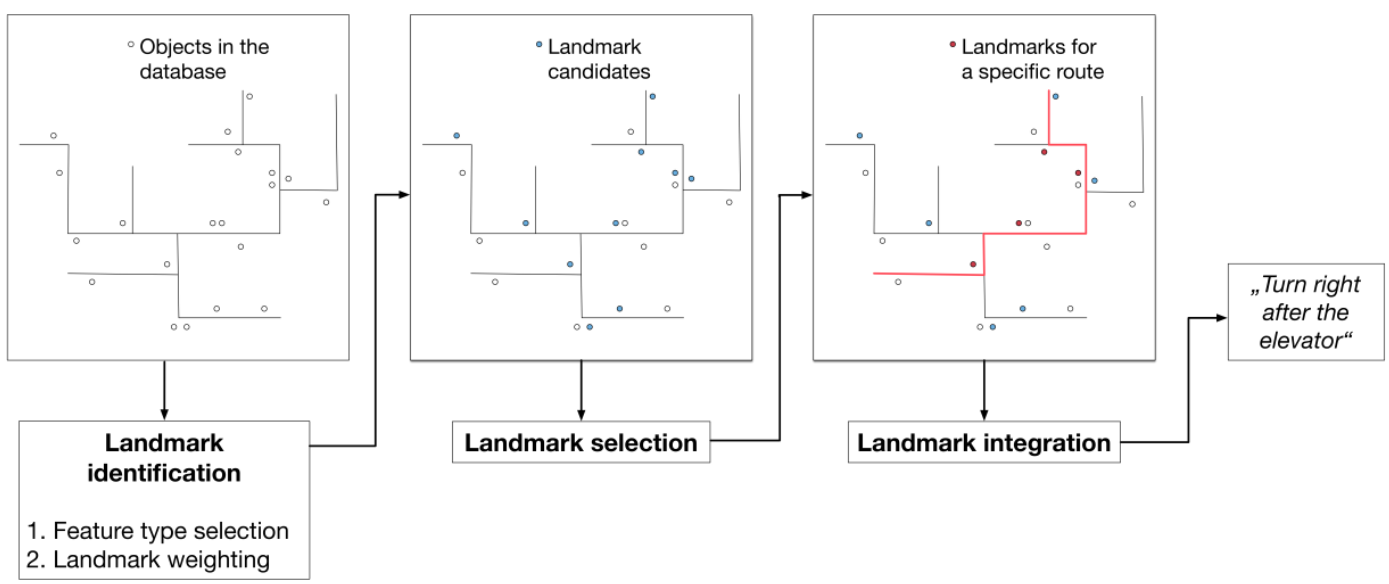

Figure 1: Overview of the indoor landmark navigation model

The ILNM is based on the method and algorithm of the LNM by Duckham et al. (2010). The LNM consists of two components: the landmark weighting system (landmark identification') and an annotation algorithm ('landmark selection').

The landmark weighting system calculates the suitability of POI categories as landmarks. For this purpose, nine factors are defined to assign weights to the POI types according to their expected experiential salience. These factors include physical size, prominence, difference from surroundings, nighttime vs. daytime salience, proximity to road, ubiquity and familiarity, length of description, spatial extents and permanence. A group of experts have to agree on a two-dimensional ranking for each factor of a POI category: 1 . How suitable is a typical instance of a category? 2. How frequent is a typical instance in a category? In the next step, these ratings are combined into an overall suitability score. Finally, this score is normalized.

The second component of the model, the annotating algorithm, creates route instructions that include landmarks based on the weighting system. The algorithm identifies POIs that are located on the route, assigns the suitability scores from the first component to these POIs, and adjusts these scores according to the following aspects: side of the road and existence of multiple landmarks on the same route leg. In the last step, the POIs with the highest scores are selected for all decision points or route legs and incorporated into route instructions.

As the LNM is designed for outdoor navigation, several challenges arise when applying this approach to indoor navigation. These include differences in the data source, in landmark types and in landmarks characteristics, as well as specific characteristics of indoor routing networks and of indoor routing instructions.

The ILNM is designed to be used with indoor spatial databases and does not rely on a specific data model. However, it has to be possible to derive the following information from within indoor GIS databases automatically: 1. indoor objects like rooms, doors, elevators or POIs as vector features; 2 . routing network. 


\section{Landmark identification}

The first step when automatically creating route instructions with landmarks is to identify features that may serve as landmarks ('landmark candidates'), independent of a specific route. Two sub-steps are required to identify good landmark candidates:

- Definition of feature types which may be suitable as landmarks ('feature type selection')

- assessment of their suitability as landmarks ('landmark weighting').

The ILNM is not based solely on POI categories, but also on general indoor feature types and room categories, which are usually available in indoor spatial databases. As these databases generally contain a lot of different feature types, it is proposed to classify features in the first step and select only those that are easily recognizable on the route and widely available in the building(s) of interest.

In the course of the landmark-weighting process, the pre-selected feature types from the previous step are assessed for their landmark suitability. The suitability factors from the LNM are modified to meet the requirements of indoor navigation. For example, the factors 'nighttime vs. daytime salience' and 'proximity to road' are removed, as they are not applicable to indoor environments. Instead, a new factor, 'availability of a unique label', is introduced.

For the assessment of the feature type categories, the ILNM suggests the same approach as in the LNM: experts have to agree on a ranking for each landmark category according to the factors defined in a five-point rating for the two dimensions of the scoring system: expected suitability and typicality of an instance of the feature category.

After all features have been rated, the overall suitability score can be determined for each category according to the landmark scoring system from the LNM. Based on these scores, the normalized weight can then be calculated. Table 1 shows an example rating.

Table 1. Example rating of the category 'elevator' of WU Campus GIS

\begin{tabular}{|l|ll|}
\hline & \multicolumn{2}{|c|}{ Elevator } \\
\hline Physical size & Suitability & Typicality \\
Prominence & Ideal & All \\
Difference from surroundings & Highly suitable & Most \\
Availability of a unique label & Highly suitable & Most \\
Ubiquity and familiarity & Never suitable & All \\
Length of description & Ideal & All \\
Spatial extents & Ideal & All \\
Permanence & Highly suitable & Most \\
\hline
\end{tabular}




\section{Landmark selection}

After having identified landmark candidates, these features can be used as a pool of potential landmarks to select from when generating instructions for specific routes within buildings. The landmark selection process uses an algorithm, which selects landmarks for a specific route according to different criteria. In the course of the selection process, the normalized suitability score of landmarks on the route is adjusted using various factors. The criteria and factors from the LNM are modified and extended in the ILNM as route paths in outdoor environments differ considerably from paths within buildings.

For instance, in indoor environments a route might lead directly through landmarks, like doors or a particular room type. In addition, the location of landmarks at the decision point and on route legs is also considered. In the case of multiple landmarks on the same route leg, numerical chunking is proposed.

\section{Landmark integration}

The last step in the automatic generation of a route description is the integration of the selected landmarks into advance wayfinding instructions. Again, the ILNM integration algorithm is adapted to the requirements of indoor navigation, e.g. by using path types instead of street names, by considering paths through open spaces and changes of floor level.

\section{Case Study}

In order to evaluate the ILNM, it was applied to the indoor spatial database of the WU Campus GIS, by means of a simulation of a real implementation, i.e. by applying the rules and algorithms stepwise and manually, partly with the help of the GIS software 'ArcGIS $10.3{ }^{\circledR}$ '. However, the logic behind each step is designed to be performed automatically in order to be implemented into a real system.

The WU Campus GIS is an online map application developed to facilitate orientation on the campus. Users can search for rooms, people, organizations, POIs and the optimal route between two points. The results are displayed on the map. Each object type (e.g. room, door, POI) is of a defined geometry type and holds different additional attributes, for instance a category or a unique name.

The landmark selection and integration process are tested with the help of three sample routes at WU Campus.

\section{Landmark identification}

In the preselection process, feature types are identified according to the criteria of the feature-type selection process. In total, 21 feature types were accepted for inclusion in this process. They can be classified into three main categories:

1. basic indoor objects (e.g. doors or elevators),

2. room categories (e.g. auditoriums or meeting rooms), 
3. POI categories (e.g. vending machines or lockers).

The rating of these selected feature types is performed by four experts. After all feature categories have been rated, the overall suitability score is determined for each category, and based on these scores the normalized weight is calculated. The most highly rated landmark categories for the WU Campus are: stairs, entrance/exit, locker, elevator and toilet.

Figure 2 illustrates the selected landmarks for the first route.

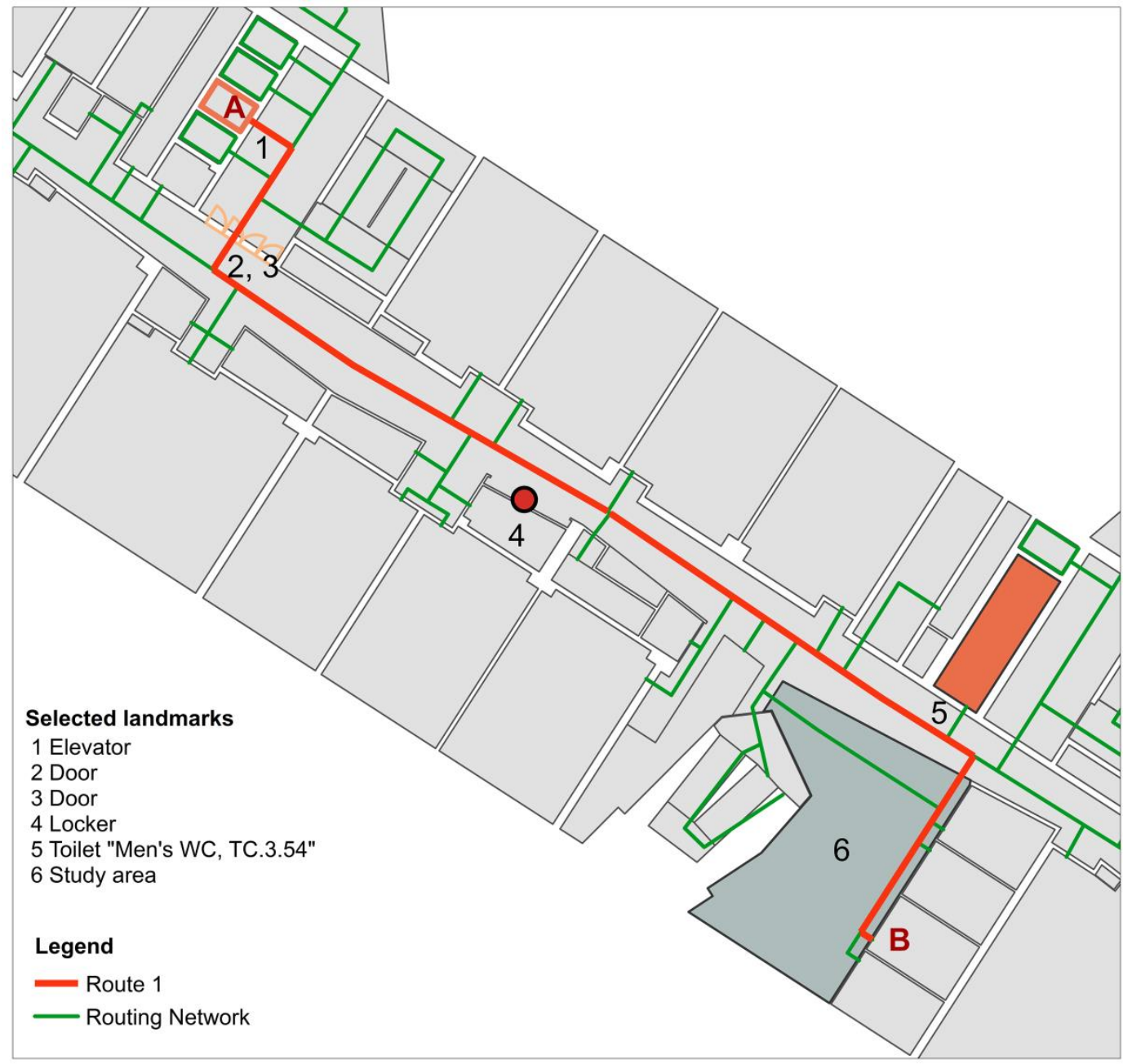

Figure 2: Selected landmarks for sample route 1 illustrated in $\operatorname{ArcGIS} \circledast$ 


\section{Landmark integration}

The last step applies the landmark integration algorithm to the selected landmarks of the previous step to generate landmark-based routing instructions. For route 1, the following instruction is generated:
1. Go along the path.
2. Turn right after the elevator.
3. Go along the path. You will pass through one door.
4. Turn left after the second door.
5. Go along the path and pass the lockers.
6. Turn right after the toilet: "Men's WC, TC.3.54".
7. Go along the path. You will pass through the study area.
8. Your destination is located on the left side of the path.

\section{Conclusion}

The generated route instructions for the three sample routes demonstrate that the ILNM algorithm generally produces correct route instructions, including landmarks.

However, the case study also shows that occasionally there might be a lack of clarity in the route instructions produced. For example, in some cases the actual visibility of landmarks on a route cannot be accurately calculated or numerical chunking might lead to ambiguous instructions.

These potential problems are primarily concerned with the main challenges of automatic indoor routing generation that were revealed in the course of the ILNM application process, i.e. the dependency on high data accuracy, the difficulty in determining the actual visibility of landmarks, and the overall complexity of indoor environments.

To overcome some of these problems, future work could examine possible enhancements both of the ILNM and of the application process of the model. First of all, the generated route instructions should be tested by humans to gain greater insights into the suitability of the selected landmarks.

Possible improvements of the ILNM include the introduction of multiple in-leg landmarks on longer route legs, the verification of previously selected landmarks, or the consideration of more complex route instructions, for instance for combining indoor and outdoor navigation. Concerning the application process of the ILNM, empirical user studies could improve parameters like the route leg threshold length for in-leg landmarks and the relative weighting changes. Finally, the ILNM could be applied to a larger set of sample routes to reveal possible additional enhancements of the model. 


\section{References}

Duckham, M., Winter, S., \& Robinson, M. (2010). Including landmarks in routing instructions. Journal of Location Based Services, 4(1), 28-52.

Elias, B. (2003). Extracting landmarks with data mining methods. In Spatial Information Theory. Foundations of Geographic Information Science (pp. 375-389). Springer.

Fellner, I. (2015). Auto-generated routing instructions with landmarks for indoor navigation. Master Thesis at the Paris Lodron-University of Salzburg.

Lovelace, K. L., Hegarty, M., \& Montello, D. R. (1999). Elements of good route directions in familiar and unfamiliar environments. In Spatial information theory. Cognitive and computational foundations of geographic information science (pp. 65-82). Springer. Retrieved from http://link.springer.com/chapter/10.1007/3-540-48384-5_5

Raubal, M., \& Winter, S. (2002). Enriching Wayfinding Instructions with Local Landmarks. In M.

Egenhofer \& D. Mark (Eds.), Geographic Information Science (Vol. 2478, pp. 243-259). Springer Berlin Heidelberg. Retrieved from http://dx.doi.org/10.1007/3-540-45799-2_17

Rehrl, K., Leitinger, S., Gartner, G., \& Ortag, F. (2009). An Analysis of Direction and Motion Concepts in Verbal Descriptions of Route Choices. In K. Hornsby, C. Claramunt, M. Denis, \& G. Ligozat (Eds.), Spatial Information Theory (Vol. 5756, pp. 471-488). Springer Berlin Heidelberg. Retrieved from http://dx.doi.org/10.1007/978-3-642-03832-7_29

Siegel, A. W., \& White, S. H. (1975). The Development of Spatial Representations of Large-Scale Environments. In H. W. Reese (Ed.) (Vol. 10, pp. 9 - 55). JAI. Retrieved from http://www.sciencedirect.com/science/article/pii/S0065240708600075

Sorrows, M. E., \& Hirtle, S. C. (1999). The nature of landmarks for real and electronic spaces. In Spatial information theory. Cognitive and computational foundations of geographic information science (pp. 37-50). Springer. Retrieved from http://link.springer.com/chapter/10.1007/3-54048384-5_3

Tom, A., \& Denis, M. (2003). Referring to landmark or street information in route directions: What difference does it make? In Spatial information theory. Foundations of geographic information science (pp. 362-374). Springer. Retrieved from http://link.springer.com/chapter/10.1007/978-3540-39923-0_24 